\title{
躍Irestice
}

\section{Uma solução para o controle do fluxo das filas de cirurgias eletivas do Sistema Único de Saúde (SUS): Sistema de Gerenciamento de Filas (SIGEFI)}

\author{
A Solution to Control the Flow of Elective Surgery Queues for the Unified Health System \\ (SUS): Queue Management System (SIGEFI)
}

\section{Simone Cristiane de Souza', Marcos Sfair Sunye ${ }^{2}$, Dante da Silva Aléoº}

\section{Resumo}

Introdução: A procura pela realização de procedimentos eletivos no Sistema Único de Saúde tem se tornado maior que a capacidade ofertada. Esta realidade torna inevitável a existência de filas, especialmente para realização de cirurgias eletivas. Na maioria dos hospitais públicos, o gerenciamento desta fila é realizado manualmente, por meio de planilhas ou agendas. Esta prática torna a fila vulnerável, passível de falhas. Objetivos: 0 objetivo deste trabalho foi diagnosticar as vulnerabilidades do manejo no controle das filas de cirurgias eletivas e apresentar uma solução por meio da elaboração de um sistema de gerenciamento usando banco de dados e linguagem de modelagem unificada. Método: Foram elaboradas modelagens dos fluxos de um sistema em tempo real, estabelecendo critérios de prioridades e gerenciamento dos dados fictícios, utilizando a teoria das filas e um protótipo de banco de dados. A inserção do paciente conforme classificação de risco foi estabelecida por meio dos protocolos da liberação anestésica. Resultado: O recurso apresentado promoveu uma significativa melhora no gerenciamento, controle e manuseio das filas de cirurgias eletivas, impactando na redução dos custos e tempo de espera para realização de procedimentos. Conclusão: O sistema de gerenciamento de filas garante a integridade e acessibilidade das filas, facilitando a manuseabilidade das mesmas.

Palavras-Chaves: Controle de Filas, Cirurgias Eletivas, Linguagem de Modelagem Unificada, Banco de Dados.

\section{Abstract}

Introduction: The search for performing elective procedures in the Unified Health System has become greater than the capacity offered. This reality makes queues inevitable, especially for elective surgeries. In most public hospitals, this queue is managed manually through spreadsheets or schedules. This practice makes the queue vulnerable, failable.

\footnotetext{
1 Mestre. Universidade Federal do Paraná. E-mail: simone.csouza@hc.ufpr.br

2 Doutor. Universidade Federal do Paraná. E-mail: ds17@inf.ufpr.br

3 Graduando. Universidade Federal do Paraná. E-mail: sunye@inf.ufpr.br

Correspondência: Hospital de Clínicas da Universidade Federal do Paraná - Rua General Carneiro, 181 - Curitiba - PR, Brasil. CEP - 80060-900.
}

RE. SAÚD. DIGI. TEC. EDU., Fortaleza, CE, v.4, n.2, p.144-162, ago./dez. 2019. 
Objectives: The objective of this work was to diagnose the vulnerabilities of management in the control of elective surgery queues, and to present a solution through the elaboration of a management system using database and unified modeling language. Method: Real-time flow modeling of a system was developed, establishing priority criteria and dummy data management using queuing theory and a database prototype. Patient insertion according to risk classification was established through anesthetic release protocols. Result: The feature presented promoted a significant improvement in the management, control and handling of elective surgery queues, impacting the reduction of costs and waiting time to perform procedures. Conclusion: The queue management system ensures the integrity, accessibility and management of queues, facilitating their handling.

Keywords: Queue Control, Elective Surgery, Unified Modeling Language, Database.

\section{Introdução}

O SUS é um dos maiores e mais complexos sistemas de saúde pública do mundo, abrangendo dos atendimentos básicos aos complexos, garantindo o acesso integral, universal e gratuito para toda a população do país ${ }^{1}$. Não obstante a excelência teórica da legislação, é de conhecimento público que na prática, o acesso aos serviços de saúde permanece um dos problemas mais graves de nossa sociedade ${ }^{2}$. Grande parte do atendimento do SUS está relacionado à procura por realização de procedimentos cirúrgicos, que são os geradores das enormes filas. Os mesmos podem ser classificados quanto à urgência cirúrgica, definidos como cirurgia eletiva, urgência e emergência. A cirurgia eletiva refere-se ao tratamento cirúrgico proposto, mas cuja realização pode aguardar ocasião mais propícia, ou seja, pode ser programado a longo prazo $^{3}$. Esta programação não está sistematicamente estruturada nos hospitais do SUS, causando um descontrole do processo de filas.

Em 2011, por meio da Lei n. o 12.550, a Empresa Brasileira de Serviços Hospitalares (EBSERH), de caráter público, vinculada ao Ministério da Educação (MEC) ${ }^{4}$, assume uma proposta de gestão inovadora e novas tecnologias. Buscando alinhar essa nova proposta de gestão e focando em garantir a execução de novas tecnologias, foi realizado um estudo dos problemas relacionados à saúde da mulher. Nesta análise, foi revisto o comportamento da rede de pactuação "Tri Partite" governo, estado e município, desde o encaminhamento da unidade básica até a realização do procedimento ${ }^{5}$. Este trabalho foi direcionado para filas de um único setor, buscando centralizar dados para desenvolvimento de uma estrutura menos vulnerável e com um número menor de elementos. O número de cirurgias eletivas na área estudada teve um aumento de $39,1 \%$ em oito meses no ano de 2017, mais de 804.961 solicitações segundo o relatório de monitoramento emitido pelo SUS no mesmo ano ${ }^{6}$. No 
entanto, após uma busca detalhada pelo governo federal, foram constatadas algumas inconsistências, havia 667.014 pacientes em fila aguardando cirurgias eletivas no mesmo período ${ }^{7}$. Essas inconsistências foram determinantes para motivação do estudo, identificando as falhas no gerenciamento de filas e a vulnerabilidade do processo de segurança da informação. Valedestacar a importância da preservação dos princípios básicos da segurança da informação: confidencialidade, integridade e disponibilidade $^{8}$. A garantia desses princípios não ocorre no formato atual.

O processo de inclusão em fila para intervenção cirúrgica acontece por meio do encaminhamento do paciente pela rede da atenção básica à instituição hospitalar pretendida. Após ser avaliado pelo médico da Unidade Básica de Saúde (UBS), e o mesmo definir a possibilidade de indicação cirúrgica, o paciente é encaminhado para a especialidade de um hospital $^{5}$. No hospital, após avaliação e definição do médico especialista quanto ao procedimento, o paciente é inserido na fila de espera. Todo o processo é realizado manualmente por meio do preenchimento de formulários e planilhas. Os pacientes, cuja conduta for o plano cirúrgico, são inseridos em fila de espera por ordem de data da consulta sem qualquer critério de prioridade ou classificação de risco.

Outro fato importante é que, para a realização do procedimento cirúrgico, os pacientes precisam passar por exames pré-operatórios. É realizada uma avaliação com o especialista que fará a liberação anestésica (LA). A avaliação pré-anestésica (APA) é uma consulta médica e especializada que deve ser efetuada previamente ao ato cirúrgico ${ }^{9}$. As liberações anestésicas (LAs) possuem prazo de validade, isto faz com que o paciente precise repetir todo o processo caso seu procedimento não ocorra a tempo, retornando para nova consulta ${ }^{9}$.

O objetivo deste estudo foi diagnosticar as vulnerabilidades do manejo no controle de filas de cirurgias eletivas, apresentando uma proposta de sistematização do gerenciamento por meio de Banco de Dados e Modelagem de Linguagem Unificada. Os objetivos específicos foram: levantar os dados estatísticos para diagnóstico das filas; criar uma ferramenta para o controle do fluxo das filas de cirurgias eletivas e implantar classificação de risco por meio da liberação anestésica. Para embasar o desenvolvimento desta proposta e manter a confidencialidade das informações das pacientes, foram utilizados apenas dados estatísticos e fictícios. A base de dados teve como casuística 600 pacientes fictícios, onde foram relacionados o tempo de espera para realização do procedimento, perdas por vencimento das LAs ou por falha no processo de recrutamento. Também foram realizados levantamentos dos custos operacionais 
para efetivação do procedimento por paciente e das perdas por realização de exames repetidos, assim como casos de agravamento clínico. Vale destacar que, apesar do estudo ter sido feito em cirurgias da saúde da mulher, o sistema se aplica para qualquer fila de cirurgias eletivas.

Foi elaborado um projeto em linguagem de modelagem unificada (UML), desenvolvendo um controle de filas, baseado na teoria das filas ${ }^{10} \mathrm{e}$ sistema em tempo real ${ }^{11}$. O gerenciamento organizado por meio da UML preconiza a implantação e controle baseado na teoria das filas, garantindo a acessibilidade e transparência das mesmas, apresentando uma perspectiva melhor quanto à redução no tempo de espera. A proposta foi chamada de sistema de gerenciamento de filas (SIGEFI) e foi desenvolvida dentro da universidade, possibilitando a futura implantação dentro de um hospital do SUS. A ideia do projeto foi baseada na vivência da realidade, usando como amostragem dados fictícios de filas de cirurgias das especialidades do cuidado da mulher.

O banco de dados (BD) foi elaborado para alinhamento das informações das pacientes, estabelecendo critérios de prioridades baseados nos prazos das LAs, conforme determinação da Sociedade Americana de Anestesiologia (ASA) ${ }^{9}$. Seguindo a teoria das filas e considerando que os critérios de prioridades específicos sejam o vencimento das LAs, seguidos da ordem de chegada, foi implementado um protótipo digital, utilizando BD e scripts automatizados $^{12}$. Essas ferramentas cuidam da gerência das filas, garantindo a segurança dos dados durante as transições de pacientes. Entre as diferentes filas, demonstram em forma gráfica a porcentagem de tratamentos concluídos e tratamentos repetidos e que, por fim, demonstram a posição do paciente em fila durante o processo ${ }^{12}$.

\subsection{Revisão da Literatura}

A saúde é direito de todos e dever do estado, assim está descrito no artigo 196 da Constituição Federal de $1988^{13}$. Além da carta magna, uma lei orgânica 8080 , de 1990, complementa com as diretrizes do SUS, que garante o acesso universal da população aos serviços de saúde $^{14}$. O SUS envolve, além das diversas instâncias do estado brasileiro, as esferas do poder executivo. Para fazer jus ao termo sistema, respeitando uma autonomia de cada ente federativo do SUS, é exigido o chamado "pacto interfederativo da Gestão Executiva"15. Com o acordo, o estado começa a revelar a grande demanda de pacientes que buscam atendimento dentro do SUS. Acredita-se que a formação de filas se concretiza em todas as instâncias, especialmente para cirurgias eletivas, pois é improvável (ou mesmo impossível) um sistema público onde não exista a formação de filas ${ }^{16}$. 
A abordagem matemática sobre a teoria das filas teve início em 1908, em Copenhague, Dinamarca, com A. K. Erlang, considerado o pai da teoria das filas. Quando trabalhava em uma companhia telefônica, realizou um estudo sobre o congestionamento das linhas telefônicas, porém, somente a partir da segunda guerra mundial ela foi aplicada a outros problemas de filas ${ }^{17}$. Para Lovelock e Wright, uma fila é a representação de uma linha de pessoas, veículos, objetos físicos ou intangíveis que aguardam sua vez de serem atendidos ${ }^{18}$. Conforme estudos realizados, Gianesi e Corrêa afirmam que, para os clientes, a sensação de espera é mais importante na formulação de sua percepção do que o tempo real gasto para esperar o serviço ${ }^{19}$. Segundo Dávalos, a teoria parte do seguinte pressuposto: "a formação de filas excede a capacidade de fornecer determinado serviço" e envolve o estudo matemático utilizando ferramentas de tratamento estatístico ou estocástico ${ }^{20}$.

Outra definição de Bruns et al. diz que: uma fila é caracterizada por um processo de chegadas (pessoas, veículos, trens etc.) a um sistema de atendimento formado por uma ou mais unidades de serviço ${ }^{21}$. As filas se classificam em: fila única e um servidor, fila única e múltiplos servidores em paralelo, múltiplas filas e múltiplos servidores em paralelo, fila única e múltiplos servidores em série. A disciplina da fila define qual a ordem em que os usuários serão atendidos ${ }^{22}$.

O problema da fila de espera é muito pouco abordado pela comunidade médica e científica. Talvez a falta de interesse seja por parecer tratar-se de uma discussão que não pertence aos meios acadêmicos e sim às instâncias governamentais ${ }^{23}$.

Pesquisadores da Universidade Federal de Santa Catarina - UFSC desenvolveram um software com dados sobre pacientes que aguardam cirurgias eletivas e um método de seleção com dados objetivos para definir quem deve ter prioridade no atendimento ${ }^{24}$. Outro modelo de gestão de filas trata-se do sistema desenvolvido pelo Hospital Universitário da Universidade Federal do Maranhão HU-UFMA, projeto que dentro do seu descritivo teórico se assemelha ao proposto $^{25}$. O sistema de gestão de filas do HU-UFMA é desenvolvido por meio da web, ferramenta utilizada para desenvolvimento de sites na Internet ou rede interna. O objetivo principal é estabelecer critérios para organização e priorização dos pacientes na fila de espera para realização de procedimentos cirúrgicos ${ }^{25}$.

Para construção do SIGEFI, foram analisados os principais aspectos dos problemas das filas, dentre eles o fato do controle ser realizado manualmente. $\mathrm{Na}$ busca por uma solução para o controle do fluxo dessas filas, foi utilizado uma estrutura mais informatizada e menos 
suscetível a falha por meio de UML e BD. Buscamos realizar um diagnóstico por meio das ferramentas utilizadas na teoria das filas ${ }^{26}$. Segundo Andrade, o principal objetivo da mesma é "avaliar o comportamento de um sistema de filas, considerando o tempo médio de permanência e de espera de um cliente ${ }^{27}$. Mesmo que os modelos matemáticos não consigam ser precisos na explicação dos fenômenos da formação de filas, ainda assim é possível dimensionar adequadamente instalações, processos e equipamentos $^{27}$. A maioria das disciplinas comuns que podem ser observadas na vida diária é First In First Out (FIFO), ou seja, o primeiro a chegar é o primeiro a ser atendido ${ }^{28}$.

No estudo, foi possível identificar os benefícios do modelo de filas, assim como verificar algumas simulações em outras áreas, que não às de saúde, podendo criar outras metodologias aplicadas. Porém, para os casos analisados de filas para realização de procedimentos eletivos em saúde da mulher, vários aspectos foram considerados, dentre eles a necessidade de se estabelecer critérios de prioridade, um deles seria a LA.

Para todos os casos, os pacientes são classificados de acordo com a escala da American Society of Anesthesiologists (ASA. Classificação do estado físico com a escala da ASA: ASA I - Paciente saudável; ASA II - Paciente com doença sistêmica leve ou moderada, sem limitação funcional; ASA III - Paciente com doença sistêmica severa, com limitação funcional; ASA IV - Paciente com doença sistêmica severa, representa risco de vida constante; ASA V - Paciente moribundo com perspectiva de óbito em 24 horas, com ou sem cirurgia; ASA VI - Paciente com morte cerebral, mantido em ventilação controlada e perfusão, para doação de órgãos (transplante). Quando o procedimento é considerado de emergência, acrescentar "E" à classificação $\mathrm{ASA}^{9}$.

\subsection{Gestão de filas}

A pactuação com o SUS, realizada pelas comissões intergestores dos seus aspectos operacionais, administrativos, financeiros e assistenciais, envolve as esferas federais, estaduais e municipais. As liberações e encaminhamentos das pacientes são realizados pela Secretaria Municipal de Saúde (SMS), por meio do código de transação. A Figura 1 representa o andamento do processo de encaminhamento da atenção básica até a realização do procedimento.

Figura 1 (nos anexos)

Todo o processo desde a avaliação do paciente, inclusão e realização do procedimento tem um custo estipulado no contrato de repasse financeiro com 0 SUS. Quando há necessidade de repetir o processo pelo vencimento das LAs ou pela demora, o SUS é onerado. 


\section{Métodos}

Não foi realizado o processo de autorização pela Instituição e submissão ao Comitê de Ética em Pesquisa em Seres Humanos (CEP), órgão regulatório que solicita a autorização dos responsáveis para liberação das informações, pois não foram utilizados dados confidenciais de pacientes ou da instituição.

Foram analisados dados de 600 pacientes fictícios, focando nos critérios de inclusão e o tempo de espera, desde o encaminhamento da rede até a realização do procedimento. Também foram estabelecidos os critérios de exclusão por fatores externos, por exemplo, óbitos e eventual realização do procedimento em outro local. Para estimativa de perdas financeiras e sociais, foram realizados os dados de liberações anestésicas vencidas. O SIGEFI foi abordado por meio de um projeto em UML, controle de filas baseadas em Teoria das Filas e sistema em tempo real ${ }^{30}$.

Baseado no fluxo de entrada dos pacientes em fila, foi desenvolvido um projeto para visualizar o controle das filas por meio da UML e a estrutura de suporte dos elementos, com simulação dos dados necessários para cadastro da paciente e descrição do procedimento. Foram desenhados os fluxos de um sistema em tempo real com critérios de prioridades apresentando a construção de um sistema baseado em teoria das filas como uma tentativa de mitigar possíveis vulnerabilidades das filas. A modelagem em UML foi usada para formalizar a proposta de uma solução para o controle de filas. Foram utilizados os seguintes diagramas: Diagrama de Caso de Uso e Diagrama de Colaboração para identificar os dados e o comportamento do paciente em fila. Diagrama de Objetos e Diagrama de Classes para definir seus interrelacionamentos. Diagrama de Atividades e Diagrama de Sequência para definir o andamento da fila ${ }^{30}$. A fila regida pela regra FIFO (atendimento na ordem de chegada, primeiro a chegar é o primeiro a sair) e buffer infinito com prioridade, conforme descrito na figura dois.

Figura 2 (nos anexos)

A proposta foi idealizar a estrutura de suporte dos elementos, com simulação dos dados necessários para cadastro da paciente e descrição do procedimento. Foram elaborados fluxos de um sistema em tempo real com critérios de classificação de risco apresentando a construção de um sistema baseado em teoria das filas. Os critérios de vencimento das LAs para inserção em fila e prioridades na realização dos procedimentos foram estabelecidos conforme determinação da ASA.

Para que o processo de fila fosse dinâmico, consequentemente foram estabelecidos três critérios de prioridades, conforme demonstrado na Figura 3. No experimento foi realizada 
uma simulação do comportamento da fila com critérios de prioridade, estabelecendo uma classificação de risco baseada na ASA da LAs. Os critérios prédefinidos foram: ASA III - vermelho - três meses de validade; ASA II Amarelo - seis meses de validade; ASA I - verde - um ano de validade.

Figura 3 (nos anexos)

Foi possível simular o número de procedimentos $\mathrm{x}$ tempo de $\mathrm{LA} \mathrm{x}$ tempo de espera, determinando 0 fator para os critérios de classificação. Na definição do percentual, foram simuladas cirurgias utilizando os critérios definidos na LA, ASA I, ASA II e ASA III. Após estudar o comportamento da fila e os casos de prioridade, foi modelado o fluxo de entrada e saída respeitando a ordem de chegada e os casos de emergência. A Figura 4 ilustra a modelagem final do fluxo das filas. As informações dos pacientes serão subtraídas do BD, cuja classificação será automática, baseada na data de vencimento da $L A$.

Figura 4 (nos anexos)

\subsection{Protótipo de Banco de Dados}

A metodologia da implementação desse protótipo foi dividida em três partes, sendo elas: a) implementação de um banco de dados que mantém todas as tabelas e campos necessários para o melhor armazenamento dos dados dos pacientes e gerenciamento das filas; b) criação de scripts que irão automatizar de forma eficiente e segura o controle das filas; c) geração automatizada de gráficos que irão mostrar de forma transparente informações sobre as cirurgias realizadas e não realizadas, além de demonstrar de forma atualizada a posição em que 0 paciente se encontra na fila.

\subsubsection{Desenvolvimento da Estrutura}

O primeiro passo da implementação desse protótipo foi a criação de um BD onde serão armazenadas todas as tabelas que contêm todas as informações necessárias para o gerenciamento eficiente das filas. O sistema de gerenciamento de BD escolhido para o projeto foi o PostgreSQL, que é um sistema relacional cujos dados estão organizados em forma de tabelas (linhas e colunas) e suas relações (chaves estrangeiras) ${ }^{31}$.

O PostgreSQL mostrou-se uma ferramenta ideal para lidar com esse problema, visto que é um sistema robusto e possuiu muitos recursos, como a execução de queries complexas, integridade transacional ${ }^{32}$. Existem diversos dados, operadores e funções oferecidas pelo próprio sistema. Também há extensões orientadas a objeto, com tipos abstratos de dados, herança e atributos multivaluados, além de apresentar a integração com diversas linguagens de programação. Em relação à segurança dos dados, PostgreSQL possui funções para definir e manter permissões e para criptografar as comunicações entre cliente/servidor ${ }^{33}$. 
Após a estruturação do BD, foram criadas tabelas que irão de fato armazenar os dados dos pacientes. No contexto, o protótipo simulou nove tabelas com funções diferenciadas e sequenciais, que são listadas e especificadas nos descritivos numerados.

1. Tabela Paciente: contém as informações principais sobre os dados do paciente. Cada paciente tem um identificador único (PacienteRegistro), a fim de servir como chave estrangeira para outras tabelas e garantir o seu anonimato. Além disso, há um campo que especifica o estado do paciente ativo ou arquivado, com o intuito de saber se ele está atualmente em algum estágio do tratamento ou se já recebeu alta hospitalar. Caso o paciente esteja ativo, há um campo que irá dizer em qual fila se encontra.

2. Tabela Diagnóstico: contém as diagnóstico do paciente, usando como chave o identificador único do mesmo (PacienteRegistro). Nela se encontram o CID, o nome do procedimento que será realizado e, logo após, a liberação dos exames pré-operatórios.

3. Tabela FilaGeral: Funciona como uma "porta de entrada" do hospital. Todos os pacientes ativos irão permanecer nesta fila até que recebam alta hospitalar. $O$ principal campo dessa fila é o LiberacaoExames, que indica se 0 paciente já passou pelas etapas de consultas e exames e está com todos os requisitos necessários para ir para a Fila de Espera. Esse campo possui três valores: zero: paciente sem consultas/exames liberados; um: paciente liberado para realizar uma cirurgia; dois: paciente liberado para tratamento clínico. Caso o campo LiberacaoExames esteja com o valor um, as informações do paciente serão movidas para a FilaEspera.

4.Tabela FilaEspera: é uma fila que armazena as informações de pacientes que estão esperando a liberação cirúrgica. Pode-se dizer que essa é a tabela mais crucial para o projeto, pois ela irá armazenar as informações que constam a data de emissão da LA e a data de vencimento da mesma, item responsável pela classificação do paciente. O campo que controla essa fila é o LiberacaoLA: zero, não liberado e um, liberado. Caso a LiberacaoLA esteja em um, a tabela FilaEspera será atualizada com o tipo da ASA que o paciente recebeu no momento da consulta com o anestesista. Após essa identificação da $L A$, o paciente será encaminhado para a fila ASA1, ASA2 ou ASA3, dependendo do valor determinado na ASA.

\section{Tabela FilaASA1, FilaASA2,}

FilaASA3: Nessas filas é feito o controle de prioridades e perdas. A prioridade é organizada pela especificação da casa, ou seja, pelo prazo de validade da mesma: ASA 1 um ano, ASA 2 seis meses, ASA 3 três meses. Caso dois ou mais pacientes possuam a mesma data de vencimento da LA, o critério de desempate é feito por ordem de inserção em fila. $O$ controle de perdas também se realiza pelo VencimentoLA. Caso o paciente não tenha realizado sua cirurgia, controlado pelo campo Status (zero não realizado, um realizado), e a LA vencer, o paciente 
será removido da FilaASA e será inserido novamente na FilaEspera. Caso o paciente seja atendido em tempo hábil, ele será removido da FilaASA e será inserido na FilaCompletados.

6. Tabela FilaPerdas: Essa fila armazena a quantidade de pacientes que não foram atendidos a tempo. Há um campo que especifica a causa da perda: zero, o paciente veio a óbito e receberá o valor zero no campo EstadoPaciente na tabela Paciente e, obviamente, não será mais reinserido em nenhuma fila; um, o paciente não foi operado antes do vencimento da LA e terá que voltar à FilaEspera novamente. Vale ressaltar que um paciente pode estar inserido mais de uma vez nessa fila, levando em conta que esse processo pode se repetir mais do que uma vez.

7. Tabela FilaCompletados: é a fila que armazena a quantidade de cirurgias que foram realizadas no prazo estipulado. Quando o paciente é inserido nessa fila o EstadoPaciente na tabela Paciente é atualizado para zero, e ele é removido de todas as tabelas de filas em que ele se encontrava. Vale ressaltar que as tabelas FilaCompletados e FilaPerdas não são mutualmente excludentes, ou seja, um paciente pode ter passado pela FilaPerdas e depois ter obtido sucesso na cirurgia $e$ ter sido inserido na FilaCompletados. As tabelas de FilaCompletados e FilaPerdas são para fins estatísticos, com o intuito de realizar uma análise comparativa ao utilizar esse sistema de gerência automatizado e o processo manual.

Todas as tabelas citadas acima possuem quatro campos em comum, sendo eles: Datalnsercao: campo preenchido de forma automática, com a data e horário em que o dado foi inserido pela primeira vez na tabela; UsuarioResponsavel: nome do usuário que inseriu o dado pela primeira vez na tabela; HstrDataTime: data da última vez que a linha de uma determinada tabela foi alterada; UsuarioModificador: nome do último usuário que realizou alterações na tabela.

Esses campos visam manter o controle e integridade dos dados inseridos nas tabelas. Com essas informações, é possível criar logs com as datas e modificações feitas em cada linha das tabelas, criando a possibilidade de se verificar a integridade dos dados inseridos. Sabendo as informações do BD e do funcionamento de cada tabela, a segunda parte do projeto é criar scripts em python que tem como objetivo principal a gerência automatizada das filas. Para realizar esse objetivo foi criado um script distinto para cada fila existente. Existem campos dentro das tabelas que precisam ser escritos manualmente por dependerem de informações médicas e que, dependendo da informação inserida/modificada, irão triggar os scripts. Exemplos desses campos que serão inseridos manualmente são: LiberacaoExames, LiberacaoLA, Status.

Foi realizado um diagrama que demonstra qual/quais scripts serão chamados dependendo da tabela que foi 
modificada. Por exemplo, caso a LiberacaoLA de uma determinada linha seja modificada na FilaEspera para um, é trigado o script que atualizará a FilaASA com as informações do paciente que acabou de ser inserido na mesma. Todas as transferências dos pacientes entre as filas serão feitas automaticamente, sem necessidade de intervenção manual.

\subsubsection{Implementação Inicial e Prova de Conceito}

Naimplementação do protótipo foram gerados dados randômicos para simular as tabelas de pacientes e diagnósticos. A partir desse ponto o sistema começa a gerenciar as filas de forma automatizada. Logo após a criação dessas duas primeiras tabelas 0 script insere automaticamente todos os pacientes que estão com EstadoPaciente = um, na fila geral. Após fila geral estar preenchida com os dados, o script de gerência da fila de espera é acionado. É verificado o campo LiberacaoExames, e caso ele seja igual a um, o paciente é inserido na fila de espera e são gerados valores randômicos para a LiberacaoLA e ASA. Caso a LiberacaoLA seja igual a um, é calculado automaticamente o prazo de vencimento do LA. Nesse mesmo passo é atualizada a tabela de diagnóstico com o valor da ASA designada ao paciente.

A Figura 5 mostra que sequencialmente é acionado o script de gerência das filas ASA; esse script verifica se o paciente possui LA, qual o valor do campo ASA e insere o paciente na fila correspondente (ASA1, ASA2 ou ASA3). Para fins de simulação, é gerado de forma randômica o valor do campo que diz se o paciente realizou o procedimento ou não.

Figura 5 (nos anexos)

Por fim, os scripts de verificação de perdas e de tratamentos completados são ativados. Sendo o estado da realização cirúrgica em zero e a LA estiver vencida (é realizado o cálculo de VencimentoLA EmissãoLA), o script de FilaPerdas é acionado e as informações desse paciente serão inseridas na fila de perdas e o mesmo irá retornar à fila de espera, onde todo o processo se repete. Caso a realização do procedimento esteja em um, ou seja, o paciente realizou sua cirurgia, os scripts de suas informações irão para a FilaCompletados, e o paciente será removido de todas as filas em que se encontra.

\section{Resultado}

Todas as fases do estudo foram estabelecidas por meio do diagnóstico da gestão de filas. Foi realizada uma análise comparativa após simulação do comportamento da fila com a proposta aplicada. $\mathrm{Na}$ primeira evidência foi identificada uma perspectiva melhor quanto à redução no tempo de espera, conseguindo estabelecer critérios de prioridade: critério I: de três anos para um ano; critério II: de dois anos para seis meses; critério III: de um ano para três meses. 
Os custos operacionais simulados foram reduzidos, pois não houve a necessidade de repetição de exames e LA. Neste caso também poderia ser calculada uma alteração no custo por agravamento clínico, pois com a demora para realização do procedimento ele se torna mais grave, consequentemente é maior o seu custo para o SUS. O BD reduziu o volume de dados manipulados manualmente, sendo possível rever o número de pessoas no manuseio.

Analisando o comportamento da fila nos 600 casos, também foi possível simular a redução de perdas. Essa análise contribuiu para sugerir uma possível organização do processo por meio de uma revisão periódica da fila, excluindo pacientes que fossem a óbito ou que tivessem realizado seu procedimento em outras instituições.

Durante todo o processo de desenvolvimento do SIGEFI, o BD utilizado foi sincronizado com o metabase, software que permite a geração de gráficos/tabelas quando conectado a um banco de dados qualquer. Após esse ciclo se completar com as tabelas devidamente preenchidas com os dados gerados, são emitidos gráficos com as estatísticas no metabase ${ }^{34}$.

\section{Conclusão}

Os objetivos do estudo foram concluídos por meio da apresentação de novas propostas para o controle do fluxo das filas de cirurgias eletivas do SUS. O SIGEFI propôs o desenvolvimento de uma estrutura menos vulnerável, identificando as falhas no gerenciamento de filas e a vulnerabilidade do processo de segurança da informação. Para embasar o desenvolvimento do estudo, foram analisados dados de 600 pacientes fictícios. Para as bases de dados foram analisados o tempo de espera para realização do procedimento, perdas por vencimento das LAs ou por falha no processo de recrutamento. Foram realizados levantamentos dos custos operacionais por paciente, tais como; perdas por realização de exames repetidos e casos de agravamento clínico.

O SIGEFI foi baseado na teoria das filas e sistema em tempo real, utilizando um protótipo de Banco de dados para alinhamento das informações, estabelecendo critérios de prioridades, baseados nos prazos das LAs. Com o uso da ferramenta de $\mathrm{BD}$, as filas foram gerenciadas automaticamente e de forma eficiente, reduzindo os custos e evitando a repetição de consultas e exames. Foi possível visualizar a garantia da segurança dos dados durante as transições de pacientes entre as diferentes filas.

O projeto está em sua fase inicial, porém foi comprovado que o SIGEFI, de forma automatizada, funciona como o desejado. $O$ próximo passo seria a parceria com um hospital do SUS, de 
modo a testar o sistema com dados reais. Além disso, cria-se a possibilidade de automatizar outras filas hospitalares, como a fila de atendimentos clínicos. Após isso, seria realizada uma integração dos scripts com o sistema hospitalar, para que todo esse processo possa acontecer de forma interna e instantânea, sem necessidade de depender da utilização de outros softwares e com a vantagem de se obter as alterações das filas em tempo real. Também seria possível monitorar diversos hospitais distintos e criar um sistema de georreferenciamento, mostrando graficamente em um mapa os hospitais monitorados e a gerência das filas dentro de cada um deles.

A proposta apresentada também identificou a possibilidade de um sistema funcional e transparente, com novos recursos que possam facilitar a manuseabilidade, integridade e acessibilidade das filas do SUS.

\section{Referências}

1 Governo Brasileiro - Constituição Federal. Sistema Único de Saúde. Brasília. [publicação na web]; 1988. 2001 acesso em 15 de abril de 2017. Disponível em: https://www.significados.com.br/sus. 16 2 Saúde Portal da. Departamento de Informática do SUS; 2017. [publicação na internet]; Disponível em: http://datasus.saude.gov.br/. 16, 33

3 Portal da Educação MEC. Classificação das Cirurgias; 2013. [publicação na web. Disponível em: https://www.portaleducacao.com.br/c onteudo/artigos/enfermagem/classificaca odascirurgias/23157. 49

4 Brasil, Ministério da Educação, EmpresaBrasileira dos Hospitais Universitários. Lei 12550. 15/12/2011;p. 1 - 1. [publicação na web]; Disponível em: http://www.andifes.org.br/lei- n-12550-cria-a-ebserh/.16

5 Secretaria Municipal de Curitiba. Unidade Básica de Saúde; 2000. [publicação na web]; Disponível em: http://www.pac.gov.br/infraestruturasocial-e-urbana/ubs-unidade-basica-desaude. 17

6 Ministério da Saúde. DATASUS; 2018. [publicação na web]; Disponível em: http://www2.datasus.gov. br/DATASUS/index.php.area=0201. 10, 31, 33

7 Brasil, Ministério da Saúde, Departamento de Análise de Situação de Saúde, Secretaria de Vigilância em Saúde. Uma Análise da Situação da Saúde e a Vigilância da Saúde da Mulher. Saúde Brasil 2011. 2012;1:163 82.17

\section{Cunha D. A Segurança da} Informação e a sua Importância para a Auditoria de Sistemas. Fortaleza: Unieducar Inteligência Educacional S/S Ltda - ME; 26/07/2013. Disponível em: https://semanaacademica.org.br/artigo/ seguranca-da-informacao-e-suaimportancia-para auditoria-de-sistemas. 17

\section{American Society of}

Anesthesiologists. Avaliação Pré Anestésica. Wikipédia, a enciclopédia livre.; 28/06/2016. [publicação na web]; Disponível em:

https://www.asahq.org/researchandpublications. 10, 18, 19, 25, 37

10 Prado D. Teoria das Filas e da Simulação. vol. 2. 6th ed. São Paulo: Falconi; 2017. 18,22

[11]Farines JM, da Silva Fraga J, de Olivei ra RS. Sistemas em Tempo Real.

Florianópolis: Universidade Federal de 
Santa Catarina; julho / 2000. 18

12 EDUCBA. Quatro funções importantes do sistema de gerenciamento de banco de dados na indústria; 2019. [publicação na web]; Disponível em: https://www.educba.com/databasemanagement-system/. 19 13 Brasil. Constituição (1988). Constituição da República Federativa do Brasil. 1988; [publicação na web]; Disponível em: http://www.planalto.gov.br/ccivil_03/con stituicao/ constituicaocompilado.htm. 22

14 Brasil, DF. Lei Orgânica no 8/880/90. A Lei 8080/90 instituiu o Sistema Único de Saúde, constituído pelo conjunto de ações e serviços de saúde, prestados por órgãos e instituições públicas federais, estaduais e municipais, da administração direta e indireta e das fundações mantidas pelo poder

público.19/09/1990;p.1-1. [publicação na web]; Disponível em:

https://www.portaleducacao.com.br/con teudo/artigos/enfermagem/a-lei-8080de-19-de-setembro-de-1990/4769. 22

15 Secretaria do Estado da Saúde.

Programa de Apoio e Qualificação de Hospitais Públicos e Filantrópicos do SUSParaná-HOSPISUS;2017.

[publicação na web]; Disponível em: http://www.saude.pr.gov.br/modules/co nteudo/conteudo.php?conteudo=2802. 17, 22, 33

16 Passos EJPF. Programação Linear Como Instrumento da Pesquisa Operacional. São Paulo: Atlas; 2008. 22 17 Erlang AK. Teoria das Filas. São Paulo; 1908. [publicação na web]; Disponível em: http://www.sucena.eng.br/ST/ST5_Mod5 TeoriadeFilas.pdf. 22, 24

18 Lovelock C, Wright L. Avaliação da Qualidade em Serviços: Estudo de Caso Realizado em uma Agência de um Banco de Varejo. São Paulo: Saraiva; 2004. [publicação na web]; Disponível em: http://www.ucs.br/etc/conferencias/index. php/mostraucsppga/mostrappga2013/pap er/viewFile/3632/1333. 22

19 Corrêa HL, Gianesi IGN. Gestão Estratégica de Operações de Serviço. web site da Correa \& Associados. 1995
1;1(1):1 - 14. [publicação na web];

Disponível em:

http://www.correa.com.br/biblioteca/arti gos/A03_Cladea_1995_Gestao_estrate gica_de_operacoes.pdf. 22

20 Dávalos PB. Hidroquímica do Estuário do Rio Caravelas. BA - Natal, RN; 2012. 22

21 Bruns R, Soncim SP, de Sinay MCF. Pesquisa Operacional: Uma Aplicação Da Teoria Das Filas A Um Sistema De Atendimento. Rio de Janeiro; 2001. [publicação na web]; Disponível em: http://www.abepro.org.br/biblioteca/ ENEGEP2001_TR60_0158.pdf. 23

22 Arenăles M, Morabito $\mathrm{R}$, Armentano V, Yanasse H. Pesquisa Operacional. vol. 6‥ 2nd ed. Brasil: Elsevier Editora Ltda.; 09/06/2015. 23 23Araujo Sarmento Junior KM, Tomita S, de Avila Kos AO. O Problema das filas de espera para cirurgias otorrinolaringológicas em serviços públicos. Revista Brasileira de Otorrinolaringologia. 2005 maio; v. $71(3): 256$ - 262. [publicação na web]; Disponível em:

http://www.scielo.br/pdf/rboto/ v71n3/a01v71n3.pdf. 16, 23, 29 24 FAPESC. A Plataforma FAPESC é um ambiente de interação da Fundação de AmparoàPesquisa elnovaçãodo EstadodeSantaCatarinacom pesquisadores, instituições de CT\&I e empresas inovadoras; 2018.

[publicação na web]; Disponível em: http://plataforma.fapesc.sc.gov.br/si gbi/\#. 23

25 HU-UFMA Hospital Universitário da UFMA -. Fila de Espera de Cirurgia; 2017. [publicação na web]; Disponível em: http://www2.ebserh.gov.br/web/huufma/noticia-destaque/-

asset_publisher/mUhqpXBVQ6gZ/conte nt/id/2860977/2018-02-nova-alaampliara-capacidade-o-hu-ufma-emmais-de-100-leitos. 24

26 Torres OF. Elementos da teoria das filas. Revista Administração de Empresas. 1966 July/Sept; vol.6(no.20):1 - 1. [publicação na web]; Disponível em: http://dx.doi.org/ 10.1590/S003475901966000300005. 24

27 Andrade EL. Introdução a Pesquisa 
Operacional. Rio de Janeiro: Livros

Técnicos e Científicos, Editora Ltda;

2011. [publicação na web]; Disponível

em:

https://www.estantevirtual.com.br/li

vros/eduardo-I Ieopoldino-de-

ndrade/introducao-a-pesquisa-

operacional/4040179445. 24

28 Steven G. Reducing Wait Times for

Health Care: What Canada Can Learn

from Theory and International

Experience; 2013. [publicação na web];

Disponível em:

https://www.fraserinstitute.org/stud

ies/reducing-wait-times- for-health-

care. 28

29 Secretaria Municipal de Curitiba.

Sistema de Regulação da SMS/Curitiba;

[publicação na web]; Disponível em:

http://www.saude.curitiba.pr.gov.br/. 10, 27

30 Ribeiro L. Artigo O que é UML e

Diagramas de Caso de Uso: Introdução

Prática à UML; 2012. [publicação na

web]; Disponível em:

https://www.devmedia.com.br/o-que-euml-e-diagramas-de-caso-de-uso-

introducao-pratica-a-uml/23408. 37

31 Source Forge. O que é o

PostgreSQL;2019. [publicação na

web]; Disponível em:

http://pgdocptbr.sourceforge.net/pg8

2/intro-whatis.html. 41

32 DEVMEDIA. Controle de Acesso no

PostgreSQL $9.1 ;$ 2019. [publicação na

web]; Disponível em:

https://www.devmedia.com.br/controlede-acesso-no-postgresql- 9-1/25392.

41

33 DEVMEDIA. Deixando seu Banco de

Dados Seguro e Rápido PostgreSQL;

2019. [publicação na web]; Disponível

em:

https://www.devmedia.com.br/postgres

ql-deixando-seu- banco-de-dados-

seguro-e-rapido/39175. 41

34 MetaBase. Metabase Documentation

- Visualizing results;2019. [publicação na

web]; Disponível em:

https://www.metabase.com/docs/v0.27.

1/users-guide/05-visualizing-

results.html. 49 


\section{Anexos}

Figura 1 - Fluxo de Encaminhamento da Rede, da UBS até a realização do procedimento.

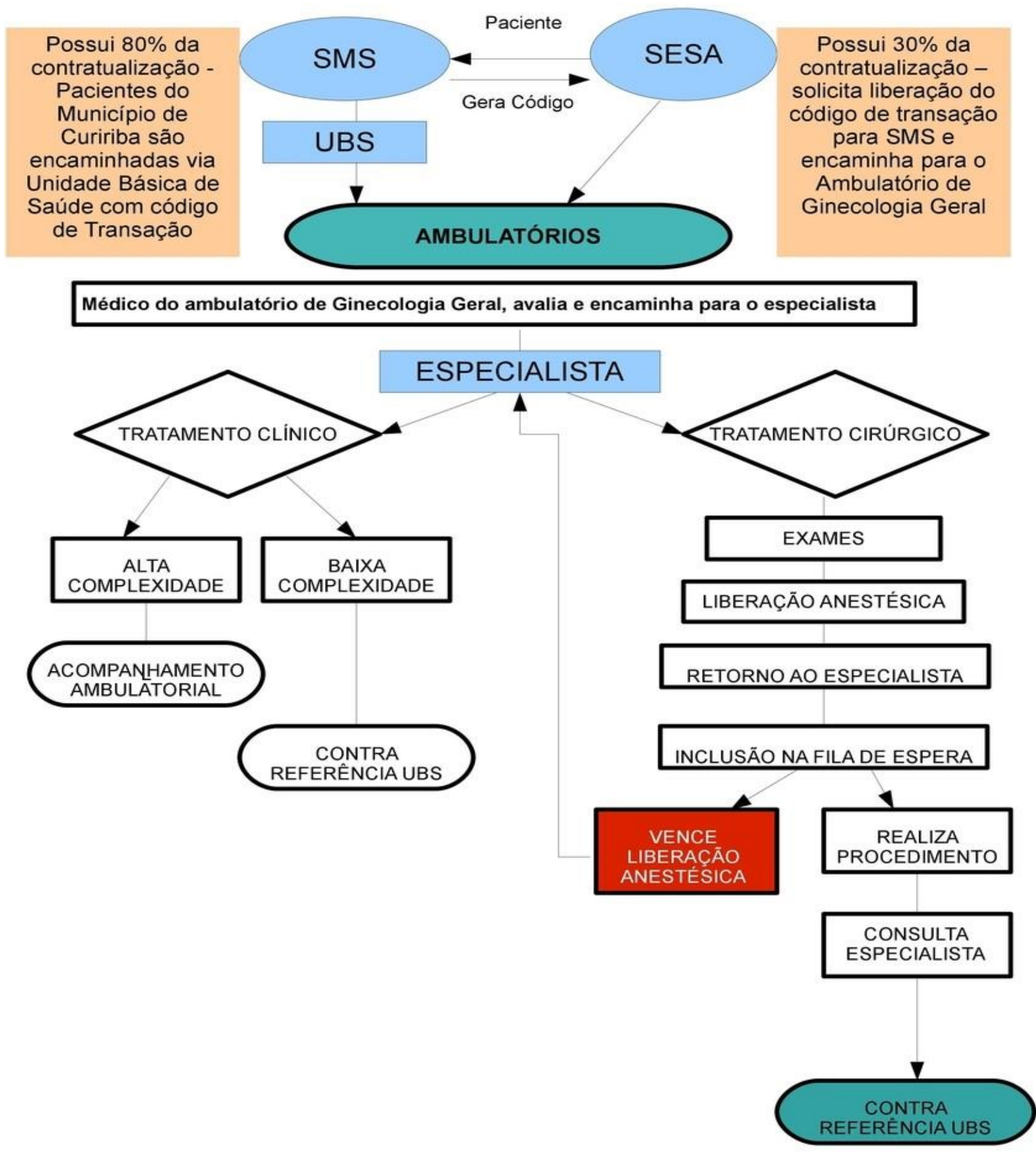

Fonte: os autores. 
Figura 2 - Demonstração do Sistema Firt In First Out - FIFO.

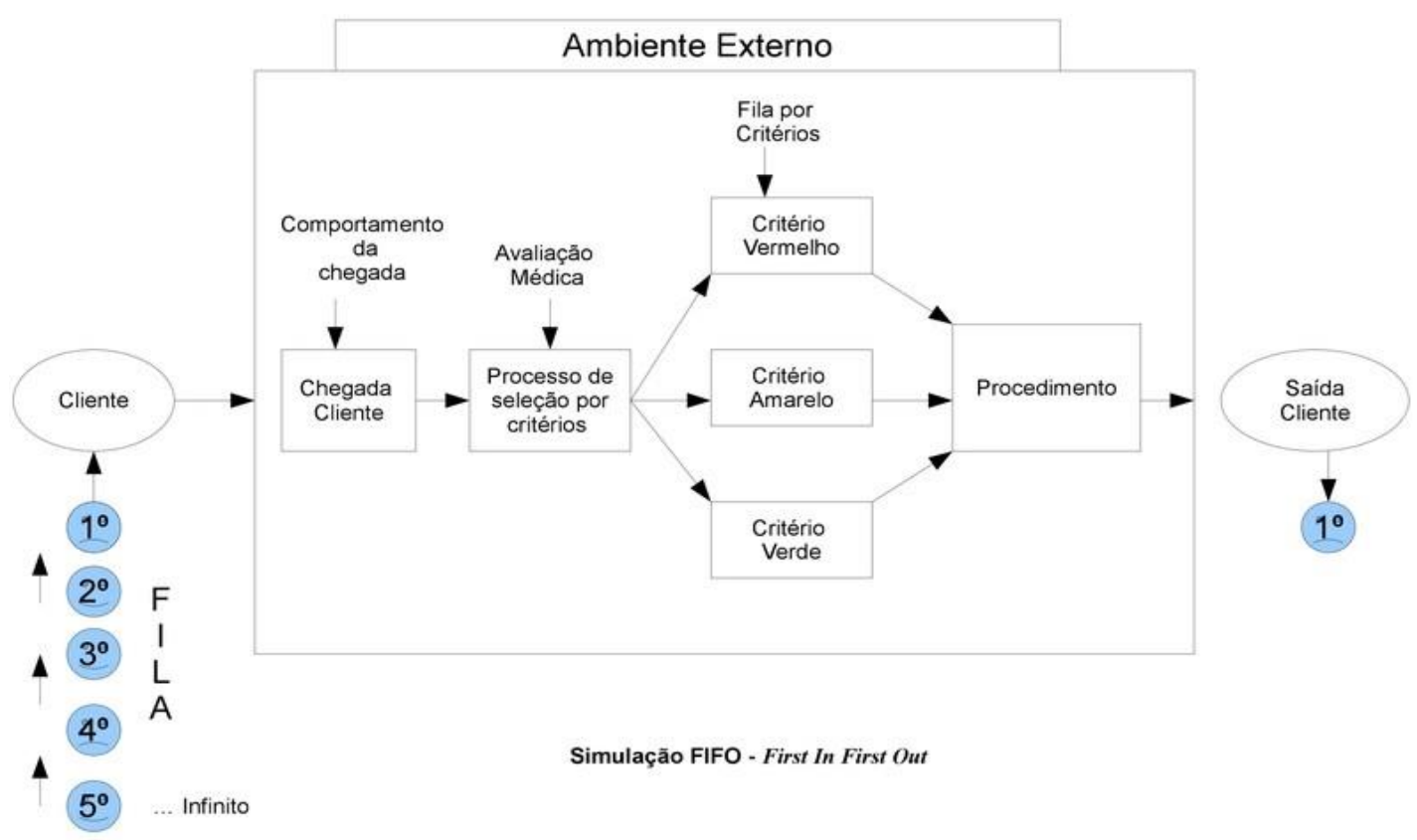

Fonte: os autores.

Figura 3 - Fluxo de Inserção em Fila por Critérios Estabelecidos pela ASA.

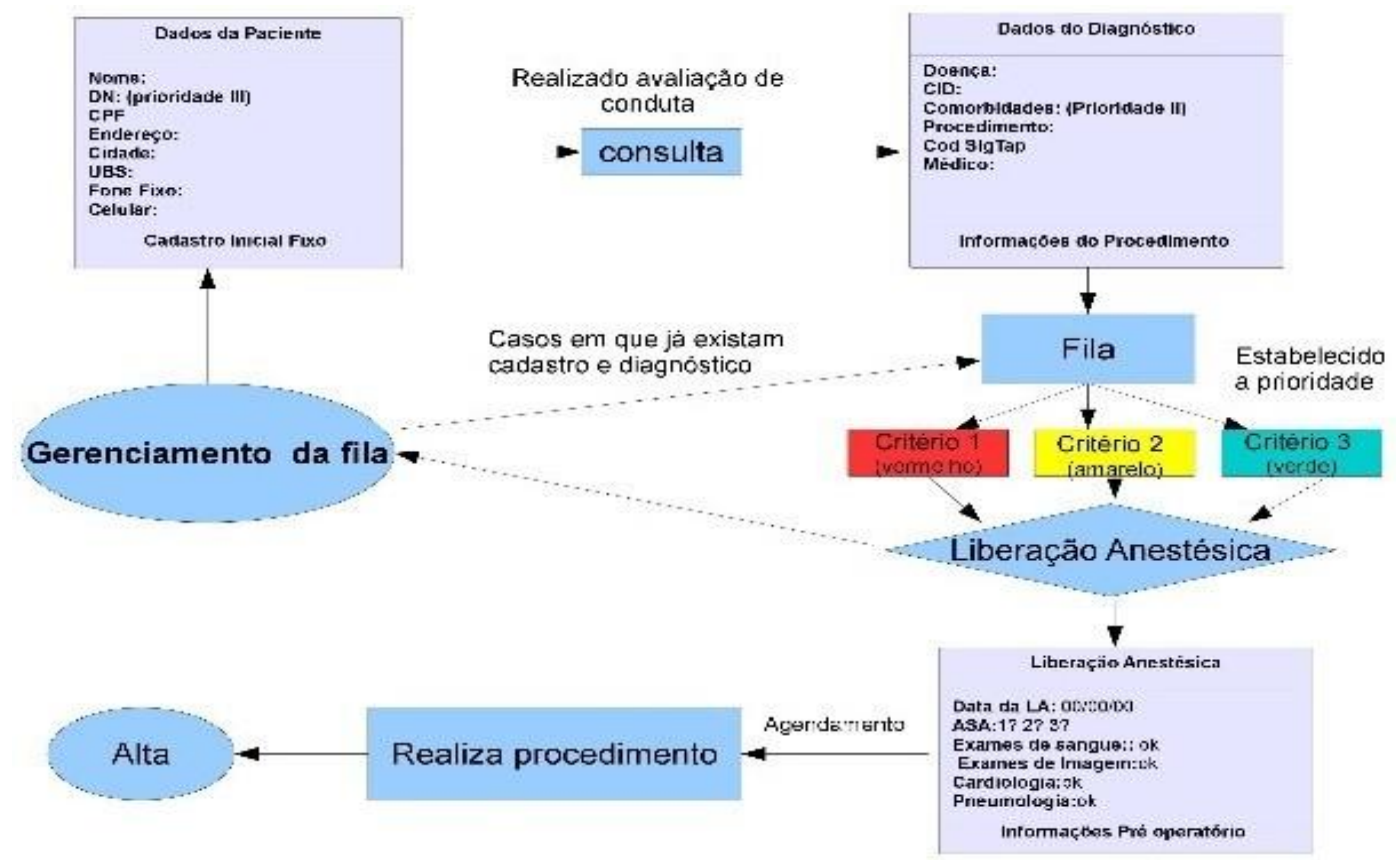

Fonte: os autores. 
Figura 4 - Modelagem do Comportamento da Fila com os Critérios Estabelecidos.

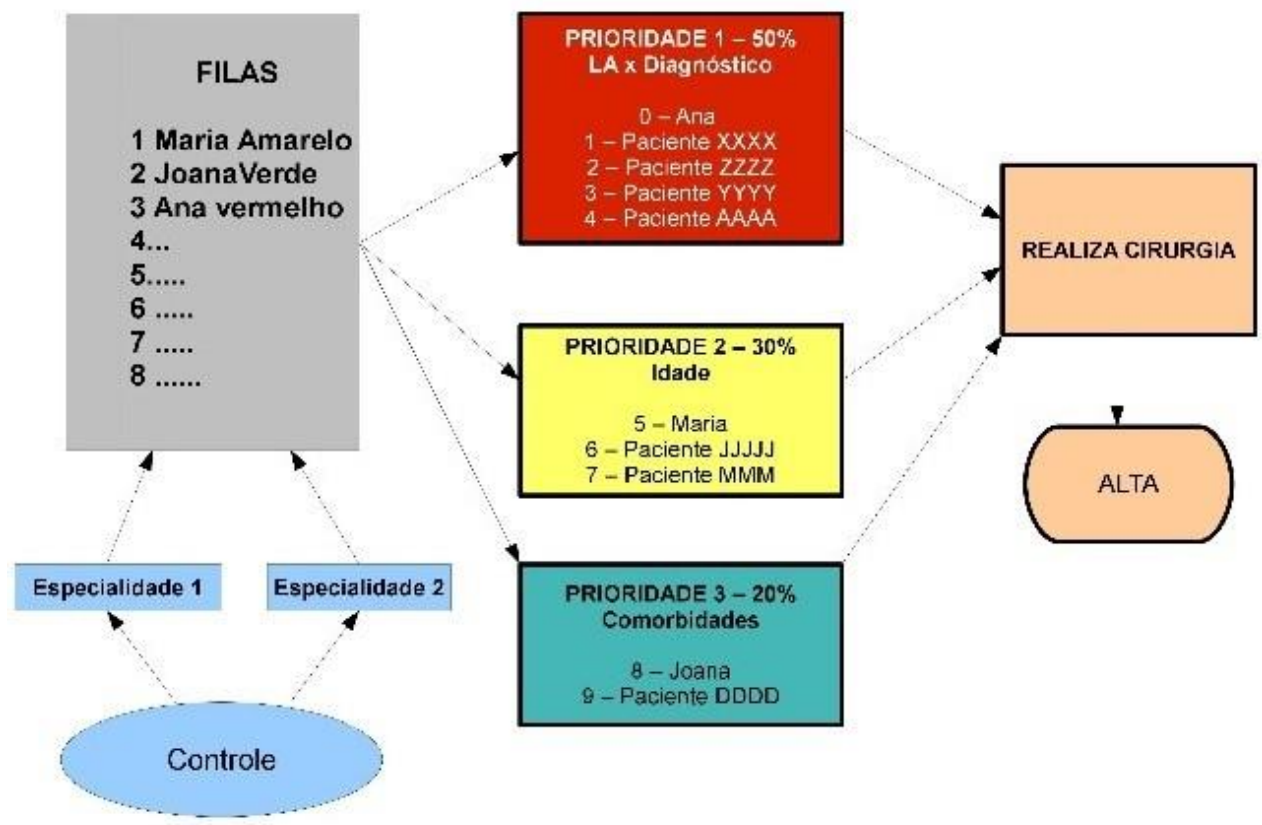

Fonte: os autores.

Figura 5 - Fluxo da Sequência das Filas.

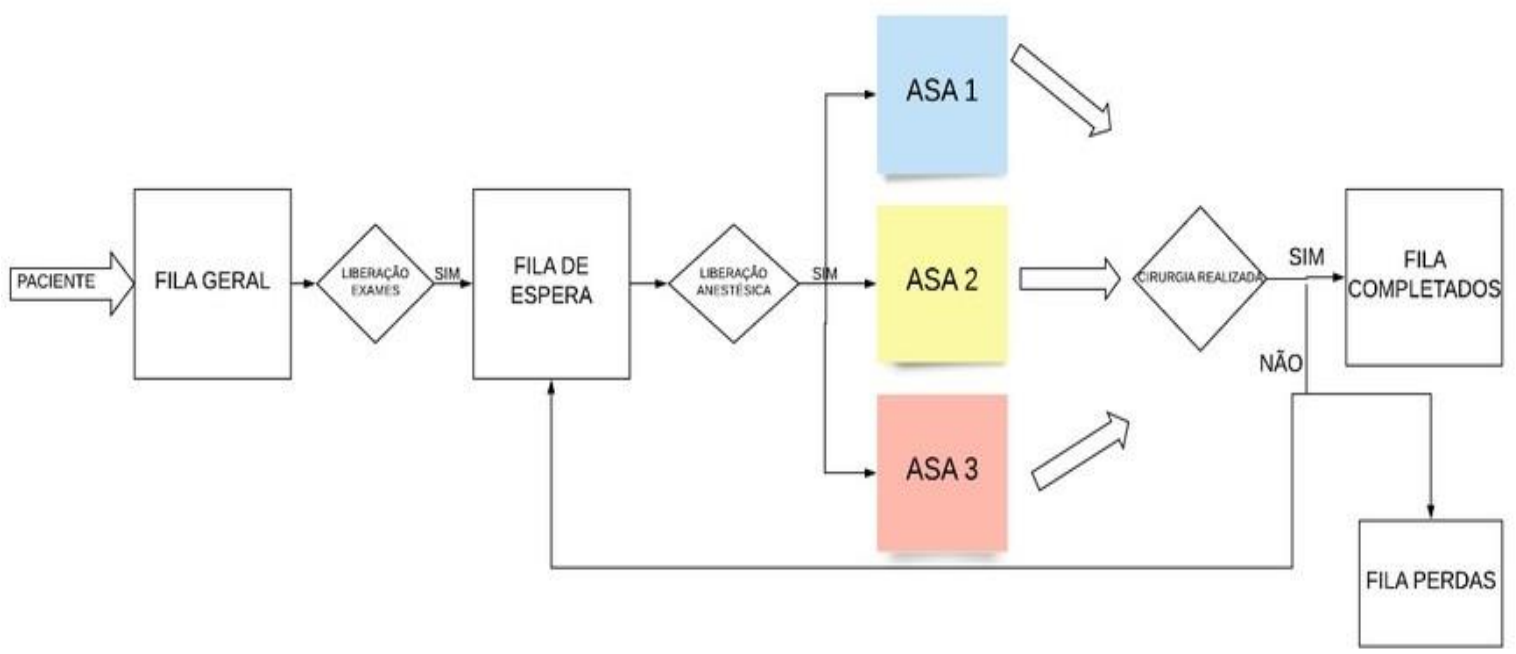

Fonte: os autores. 


\section{Como citar este artigo}

Souza SC; Sunye MS; Aléo DS. Uma solução para o controle do fluxo das filas de cirurgias eletivas do Sistema Único de Saúde (SUS): Sistema de Gerenciamento de Filas (SIGEFI). Revista de Saúde Digital e Tecnologias Educacionais. [online], volume 4, n. 2. Editor responsável: Luiz Roberto de Oliveira. Fortaleza, mês e ano, p. 144-162. Disponível em: http://periodicos.ufc.br/resdite/index. Acesso em "dia/mês/ano".

Data de recebimento do artigo: 03/10/2019

Data de aprovação do artigo: 25/11/2019 